\title{
Real Quadratic Analogs of Traces of Singular Moduli
}

\author{
W. Duke ${ }^{1}$, Ö. Imamoḡlu ${ }^{2}$, and Á. Tóth ${ }^{3}$ \\ ${ }^{1}$ UCLA Mathematics Department Box 951555, Los Angeles, CA \\ 90095-1555, USA, ${ }^{2}$ ETH, Mathematics Department CH-8092, Zürich, \\ Switzerland, and ${ }^{3}$ Eötvös Loránd University Department of Analysis, \\ Budapest, Hungary
}

Correspondence to be sent to: wdduke@ucla.edu

In this paper, regularized Petersson inner products of certain weight $\frac{3}{2}$ weakly holomorphic (or harmonic) modular forms are expressed in terms of real quadratic analogs of traces of singular moduli. The proofs make use of the theory of weight $\frac{1}{2}$ mockmodular forms.

\section{Introduction}

There are numerous connections between quadratic fields and modular forms. One of the most beautiful is provided by the theory of singular moduli, which are the values of the classical $j$-function

$$
j(\tau)=q^{-1}+744+196884 q+21493760 q^{2}+\cdots \quad(q=e(\tau))
$$

at imaginary quadratic irrationalities. (Singular moduli are also sometimes called singular invariants, for instance in [8]. Strictly speaking, this is more accurate since the term moduli refers to values of the modulus $k(\tau)$ of an elliptic integral.) It was observed recently by Kaneko, and independently by Duke et al. that it is possible to

Received May 21, 2010; Revised July 19, 2010; Accepted July 22, 2010

Communicated by Prof. Richard Borcherds

(C) The Author(s) 2010. Published by Oxford University Press. All rights reserved. For permissions, please e-mail: journals.permissions@oup.com. 
define real quadratic analogs of singular invariants through cycle integrals of the $j$ function. Although their theory is still in its infancy, various results and conjectures about them have been given in [3,5]. In this paper, we will explore them further and give some new applications of the results of [3]. In particular, we will express certain sums over classes ("traces") of these invariants as regularized inner products of weakly holomorphic modular forms of weight $\frac{3}{2}$.

\section{Statement of Results}

Unless otherwise specified, in this paper $d$ always denotes a discriminant, which means that it is a non-zero integer with $d \equiv 0,1(\bmod 4)$. It is called fundamental if it is the discriminant of the number field $\mathbb{Q}(\sqrt{d})$. Every discriminant $d$ is a unique square multiple of a fundamental discriminant. Suppose that $d$ is a non-square discriminant. For each such $d$, let $\mathcal{Q}(d)$ be the set of all complex numbers of the form

$$
w=\frac{-b+\sqrt{d}}{2 a} \text { where } d=b^{2}-4 a c
$$

with relatively prime $a, b, c \in \mathbb{Z}$. When $d<0$ we assume that $a>0$ and that $\sqrt{d} \in \mathcal{H}$, the upper half-plane. The modular group $\Gamma=\operatorname{PSL}(2, \mathbb{Z})$ splits $\mathcal{Q}(d)$ into equivalence classes through its natural linear fractional action. The set of classes $\Gamma \backslash \mathcal{Q}(d)$ forms a finite abelian group of order $h(d)$, which is the class number. The group operation can be defined through that of associated binary quadratic forms, where we associate to $w$ the form $a x^{2}+b x y+c y^{2}$. The identity class is represented by $\sqrt{d} / 2$ if $d$ is even and by $(-1+$ $\sqrt{d}) / 2$ if $d$ is odd. The isotropy group $\Gamma_{w}=\{\gamma \in \Gamma ; g w=w\}$ consists of all transformations

$$
\gamma= \pm\left(\begin{array}{cc}
\frac{t+b u}{2} & c u \\
-a u & \frac{t-b u}{2}
\end{array}\right)
$$

where $(t, u)$ is an integral solution to the Pell equation $t^{2}-d u^{2}=4$. When $d<0$ this group is trivial unless $d=-3,-4$, in which case it has order 3 or 2 , respectively. When $d>0$ it is infinite cyclic with generator in (2.1) coming from $t, u>0$ with $t$ minimal. In all cases the regulator $R(d)$, which is a certain co-volume of $\Gamma_{w}$, depends only on $d$ and is given for any $w \in \mathcal{Q}(d)$ by

$$
R(d)= \begin{cases}2 \pi\left(\# \Gamma_{w}\right)^{-1} & \text { if } d<0, \\ 2 \log \varepsilon_{d} & \text { if } d>0,\end{cases}
$$


where $\varepsilon_{d}=(t+u \sqrt{d}) / 2$. If $d \neq 1$ is fundamental, then we have the elegant class number formula of Dirichlet

$$
R(d) h(d)=|d|^{1 / 2} L\left(1, \chi_{d}\right)
$$

where $\chi_{d}$ is the Kronecker symbol. For positive fundamental $d>1$ the size of $R(d)$ is both erratic and mysterious as $d$ varies. This makes the corresponding behavior of $h(d)$ even more inaccessible than that of $L\left(1, \chi_{d}\right)$.

It is useful to define the general Hurwitz function $h^{*}(d)$ for the non-square discriminants $d$ by

$$
h^{*}(d)=\frac{1}{2 \pi} \sum_{\ell^{2} \mid d} R\left(\frac{d}{\ell^{2}}\right) h\left(\frac{d}{\ell^{2}}\right) .
$$

For $d<0$ we have that $h^{*}(d)=H(|d|)$, where $H(n)$ is the usual Hurwitz class number. By convention $H(0)=-\frac{1}{12}$ and $H(n)=0$ for $n \equiv 1,2(\bmod 4)$. Early on it was realized that there is a connection between $H(n)$ and modular forms of weight $\frac{3}{2}$. Let $\theta$ be the classical Jacobi theta function

$$
\theta(\tau)=1+2 q+2 q^{4}+2 q^{9}+\cdots,
$$

which is a modular form of weight $\frac{1}{2}$ for $\Gamma_{0}(4)$, the usual congruence subgroup of $\Gamma$. Here as usual $q=e(\tau)=\mathrm{e}^{2 \pi \mathrm{i} \tau}$ for $\tau=x+\mathrm{i} y \in \mathcal{H}$. Then

$$
\theta(\tau)^{3}=\sum_{n \geq 0} r_{3}(n) q^{n}=1+6 q+12 q^{2}+8 q^{3}+6 q^{4}+24 q^{5}+\cdots
$$

where $r_{3}(n)$ is the number of representations of $n$ as the sum of three squares. Now $\theta(\tau)^{3}$ is a modular form of weight $\frac{3}{2}$ for $\Gamma_{0}(4)$ and its coefficients are related to $H(n)$ by the famous result of Gauss, which states that for all $n \geq 0$ we have

$$
r_{3}(n)=12(H(4 n)-2 H(n))
$$

Zagier [9] (see also [4]) showed that if we allow a modular form to be harmonic we get a generating series for $H(n)$ itself. Specifically, the function

$$
g_{0}(\tau)=\sum_{n \geq 0} H(n) q^{n}+y^{-1 / 2} \sum_{n \in \mathbb{Z}} \beta\left(4 \pi n^{2} y\right) q^{-n^{2}}
$$


has weight $\frac{3}{2}$ for $\Gamma_{0}(4)$, meaning that it transforms like $\theta^{3}$. Here for $y>0$

$$
\beta(y)=\frac{1}{16 \pi} \int_{1}^{\infty} t^{-3 / 2} \mathrm{e}^{-y t} \mathrm{~d} t
$$

is an incomplete gamma function. The Fourier coefficients of $g_{0}$ are supported on integers $n$ with $n \equiv 0,3(\bmod 4)$, and there are no non-zero holomorphic forms of weight $\frac{3}{2}$ for $\Gamma_{0}(4)$ with this property. If we allow poles in the cusps, then there are infinitely many, and the space $M_{3 / 2}^{!}$of all such forms has a natural basis which is also found by Zagier [10]. Its elements are parameterized by positive discriminants (squares allowed). The first one is given explicitly in terms of the usual modular forms $E_{4}$ and $\Delta$ by

$$
g_{1}(\tau)=\theta\left(\tau+\frac{1}{2}\right) \frac{E_{4}(4 \tau)}{\Delta(4 \tau)^{1 / 4}}=q^{-1}-2+248 q^{3}-492 q^{4}+4119 q^{7}-7256 q^{8}+\cdots
$$

For each $d>0$ there is a unique form $g_{d} \in M_{3 / 2}^{!}$with Fourier series of the form

$$
g_{d}(\tau)=q^{-d}+\sum_{0 \leq n \equiv 0,3(4)} B(d, n) q^{n}
$$

where the coefficients $B(d, n)$ are all integers and

$$
B(d, 0)= \begin{cases}-2 & \text { if } d \text { is a square } \\ 0 & \text { otherwise }\end{cases}
$$

For a fixed positive $d$, Zagier discovered that $B(d, n)$ is analogous to the Hurwitz class number $H(n)$. This is illustrated by the simplest case when $d=1, n>4$ and $-n$ is fundamental where

$$
B(1, n)=\sum_{w \in \Gamma \backslash \mathcal{Q}(-n)} j_{1}(w)
$$

with $j_{1}=j-744$ being the $j$-function normalized to have constant term 0 . The fact that $B(1, n)$ is an integer reflects the classical result that $j_{1}(w)$ is an algebraic integer and the sum over $\Gamma \backslash \mathcal{Q}(-n)$ gives its algebraic trace. For every $d$ and $n$ such a formula holds for $B(n, d)$, but it involves character twists and more general modular functions.

In this paper we will consider the regularized Petersson inner product of two different $g_{d}$ 's. Recall that for two modular forms $f$ and $g$ of weight $\frac{3}{2}$ for $\Gamma_{0}(4)$ with 
singularities only in the cusps we can define

$$
\langle f, g\rangle=\lim _{Y \rightarrow \infty} \int_{\mathcal{F}_{4}(Y)} f(\tau) \overline{g(\tau)} y^{3 / 2} \frac{\mathrm{d} x \mathrm{~d} y}{y^{2}}
$$

where $\mathcal{F}_{4}(Y)$ is the standard truncated fundamental domain for $\Gamma_{0}(4)$ obtained by removing $Y$-neighborhoods of the cusps and given explicitly below. Of course this might not converge but when it does its value can be interesting.

Theorem 2.1. For $d>1$ fundamental, and $g_{0}$ and $g_{d}$ defined in (2.5) and (2.7) we have

$$
\left\langle g_{d}, g_{0}\right\rangle=-\frac{3}{4 \pi} d^{-1 / 2}\left(\log \varepsilon_{d}\right) h(d)
$$

In view of the class number formula (2.2) this can be written as

$$
L\left(1, \chi_{d}\right)=-\frac{8 \pi}{3}\left\langle g_{d}, g_{0}\right\rangle
$$

It is a pleasant surprise that the inner product of the "imaginary quadratic" functions $g_{0}$ and $g_{d}$ contains real quadratic information! Note that it follows that $g_{0}$ and $g_{d}$ are never orthogonal for $d>1$ fundamental. Theorem 2.1 should be compared with the weight $\frac{1}{2}$ result of Borcherds [1, Corollary 9.6, p. 530].

What about $\left\langle g_{d_{1}}, g_{d_{2}}\right\rangle$ when $d_{1}, d_{2}>0$ ? Looking at (2.8) for a clue, to give a formula here we first must show how to extend the domain of the modular function $j_{1}=j-744$ to include real quadratic numbers. This can be done by setting for $d>0$

$$
j_{1}(w)=j_{1}\left(\frac{-b+\sqrt{d}}{2 a}\right)=\frac{1}{2 R(d)} \int_{-\alpha}^{\alpha} j_{1}\left(-\frac{b}{2 a}+\frac{\mathrm{i} \sqrt{d}}{2|a|} \mathrm{e}^{i \theta}\right) \frac{\mathrm{d} \theta}{\cos \theta}
$$

where

$$
\alpha=2 \tan ^{-1}\left(\frac{u \sqrt{d}}{t}\right),
$$

with $t$ and $u$ defined below (2.1). As will be shown below, this extension $j_{1}$ is still $\Gamma$-invariant. It is obvious from (2.10) that for $w^{\prime}=(b+\sqrt{d}) /-2 a$ we have $j_{1}(w)=j_{1}\left(w^{\prime}\right)=$ $\overline{j_{1}(-w)}$. This observation was first made by Kaneko [5], who used a different looking but equivalent definition of $j_{1}(w)$.

Suppose that $D$ is a fundamental discriminant. It is known that the real characters $\chi$ of $\Gamma \backslash \mathcal{Q}(D)$, the genus characters, are in one-to-one correspondence with distinct 
(unordered) factorizations $D=d d^{\prime}$ of $D$ into fundamental discriminants. The value of $\chi$ can be computed unambiguously from a representative $w=(-b+\sqrt{D}) / 2 a$ of $\Gamma \backslash \mathcal{Q}(D)$ by

$$
\chi(w)= \begin{cases}\left(\frac{d}{a}\right) & \text { if }(a, d)=1 \\ \left(\frac{d^{\prime}}{a}\right) & \text { if }\left(a, d^{\prime}\right)=1 .\end{cases}
$$

Clearly $\chi(w)=\chi\left(w^{\prime}\right)=\chi(-w)$. Using the definition of $j_{1}(w)$ for real quadratic $w$ given above we have the following evaluation.

Theorem 2.2. Suppose that $d$ and $d^{\prime}$ are distinct positive fundamental discriminants. Then

$$
\left\langle g_{d}, g_{d^{\prime}}\right\rangle=\frac{3}{4 \pi} D^{-1 / 2} \log \varepsilon_{D} \sum_{w \in \Gamma \backslash \mathcal{Q}(D)} \chi(w) j_{1}(w),
$$

where $D=d d^{\prime}$ and $\chi$ is the corresponding genus character of $\Gamma \backslash \mathcal{Q}(D)$.

In particular, we have

$$
\left\langle g_{d}, g_{1}\right\rangle=\frac{3}{4 \pi} d^{-1 / 2} \log \varepsilon_{d} \sum_{w \in \Gamma \backslash \mathcal{Q}(d)} j_{1}(w)
$$

We remark that Theorems 2.1 and 2.2 can be generalized to include some nonfundamental discriminants.

\section{Preliminaries}

First we will review some basic facts about modular forms. Recall the Jacobi theta function $\theta(\tau)$, which was defined in (2.4). Set

$$
j_{\theta}(\gamma, \tau)=\frac{\theta(\gamma \tau)}{\theta(\tau)} \quad \text { for } \gamma \in \Gamma_{0}(4)
$$

As usual, for non-zero $z \in \mathbb{C}$ and $v \in \mathbb{R}$ we define $z^{v}=|z|^{v} \exp (\mathrm{i} v \arg z)$ with $\arg z \in(-\pi, \pi]$. We have the explicit evaluation [7, p. 447]

$$
j_{\theta}(\gamma, \tau)=(c \tau+a)^{1 / 2} \varepsilon_{a}^{-1}\left(\frac{c}{a}\right) \text { for } \gamma= \pm\left({ }_{c}^{*} a\right) \in \Gamma_{0}(4),
$$


where $(c / a)$ is the extended Kronecker symbol and

$$
\varepsilon_{a}= \begin{cases}1 & \text { if } a \equiv 1(\bmod 4) \\ i & \text { if } a \equiv 3(\bmod 4)\end{cases}
$$

For $k \in \frac{1}{2} \mathbb{Z}$ say that $f$ defined on $\mathcal{H}$ has weight $k$ for $\Gamma_{0}(4)$ (or just has weight $k$, when the group is clear) if

$$
f(\gamma \tau)=j_{\theta}(\gamma, \tau)^{2 k} f(\tau)
$$

for all $\gamma \in \Gamma_{0}(4)$. For $k \in 2 \mathbb{Z}$ and $\gamma=\left(\begin{array}{ll}a & b \\ c & d\end{array}\right) \in \operatorname{PSL}(2, \mathbb{R})$ it is usual to write

$$
\left(\left.f\right|_{k} \gamma\right)(\tau)=(c \tau+d)^{-k} f(\tau)
$$

and for such $k$ we see from (3.2) that $f$ has weight $k$ for $\Gamma_{0}(4)$ if and only if $\left.f\right|_{k} \gamma=f$ for all $\gamma \in \Gamma_{0}(4)$.

The group $\Gamma_{0}(4)$ has three inequivalent cusps represented by i $\infty, 0$, and $\frac{1}{2}$. Let $\mathcal{F}_{4}$ be the fundamental domain for $\Gamma_{0}(4)$ shown in Figure 1 . Let $\mathcal{F}_{4}(Y)$ be the domain truncated at cusp i $\infty$ by the $\operatorname{line} \operatorname{Im}(\tau)=Y$, at cusp $\frac{1}{2}$ by the circle $\left|\tau-\left(\frac{1}{2}+\mathrm{i} / 8 Y\right)\right|=1 / 8 Y$, and at cusp 0 by the circle $|\tau-\mathrm{i} / 8 Y|=1 / 8 \mathrm{Y}$. Consider the scaling matrices in $\sigma_{0}, \sigma_{1 / 2} \in$ $\operatorname{PSL}(2, \mathbb{R})$ given by

$$
\sigma_{0}= \pm\left(\begin{array}{cc}
0 & -\frac{1}{2} \\
2 & 0
\end{array}\right) \quad \text { and } \quad \sigma_{1 / 2}= \pm\left(\begin{array}{ll}
1 & 0 \\
2 & 1
\end{array}\right)
$$

One checks that $\sigma_{0}$ maps i $\infty$ to 0 and $\operatorname{Im}(\tau)=Y$ to $|\tau-\mathrm{i} / 8 Y|=1 / 8 Y$ and that $\sigma_{1 / 2}$ maps i $\infty$ to $\frac{1}{2}$ and $\operatorname{Im}(\tau)=Y$ to $\left|\tau-\left(\frac{1}{2}+\mathrm{i} / 8 Y\right)\right|=1 / 8 Y$, both with unchanged orientation. The next result follows easily.

Lemma 3.1. Suppose that $f: \mathcal{H} \rightarrow \mathbb{C}$ is continuous and that $\left.f\right|_{2} \gamma=f$ for all $\gamma \in \Gamma_{0}(4)$. Then for $Y \geq 2$ we have

$$
\int_{\partial \mathcal{F}_{4}(Y)} f(\tau) \mathrm{d} \tau=-\int_{-1 / 2+\mathrm{i} Y}^{1 / 2+\mathrm{i} Y}\left(f(\tau)+\left.f\right|_{2} \sigma_{0}(\tau)+\left.f\right|_{2} \sigma_{1} / 2(\tau)\right) \mathrm{d} \tau
$$

where the first integral is taken in the positive direction around the boundary of $\mathcal{F}_{4}(Y)$. 


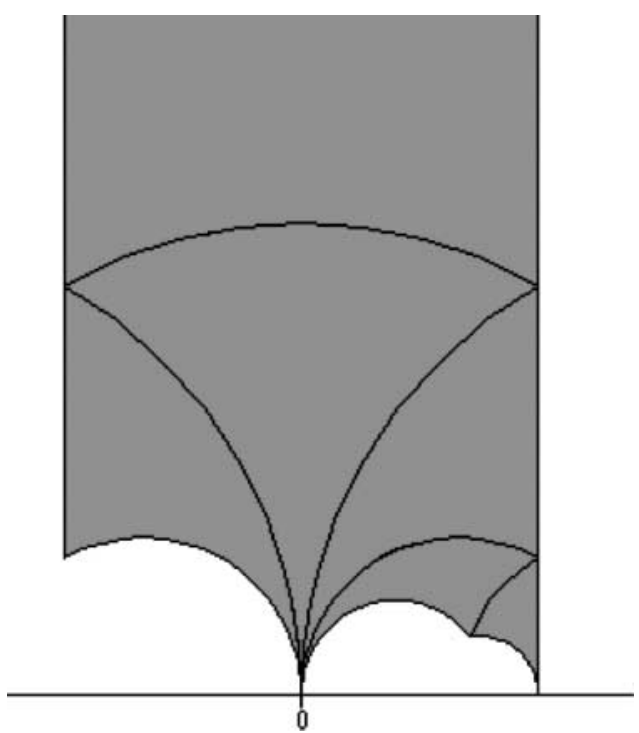

Fig. 1. The fundamental domain $\mathcal{F}_{4}$ for $\Gamma_{0}(4)$.

If $f$ of weight $k$ for $\Gamma_{0}(4)$ is smooth, for example, it will have a Fourier expansion in each cusp. For the cusp at i $\infty$ we have the Fourier expansion

$$
f(\tau)=\sum_{n} a(n, y) e(n x)
$$

which, if $f$ is holomorphic, has $a(n, y)=a(n) e(n i y)$. Set

$$
f^{\mathrm{e}}(\tau)=\sum_{n \equiv 0(2)} a\left(n, \frac{y}{4}\right) e\left(\frac{n x}{4}\right) \quad \text { and } \quad f^{\circ}(\tau)=\sum_{n \equiv 1(2)} a\left(n, \frac{y}{4}\right) e\left(\frac{n}{8}\right) e\left(\frac{n x}{4}\right)
$$

Suppose that $k \in \frac{1}{2}+\mathbb{Z}$ and that the Fourier coefficients $a(n, y)$ satisfy the plus space condition, meaning that they vanish unless $(-1)^{k-1 / 2} n \equiv 0,1(\bmod 4)$. An easy extension of arguments given in [6, p. 190] shows that such an $f$ satisfies

$$
\left(\frac{2 \tau}{\mathrm{i}}\right)^{-k} f\left(-\frac{1}{4 \tau}\right)=\alpha f^{\mathrm{e}}(\tau) \text { and }\left(\frac{2 \tau+1}{\mathrm{i}}\right)^{-k} f\left(\frac{\tau}{2 \tau+1}\right)=\alpha f^{\mathrm{o}}(\tau),
$$

where

$$
\alpha=(-1)^{\lfloor(2 k+1) / 4\rfloor} 2^{-k+1 / 2} .
$$


In particular, the behavior of such an $f$ at the cusps 0 and $\frac{1}{2}$ is determined by that at $i \infty$. Thus to check that a form is weakly holomorphic, meaning it is holomorphic on $\mathcal{H}$ and meromorphic in the cusps, one only needs to look at the Fourier expansion at i $\infty$. As is now standard, we denote by $M_{k}^{!}$the space of weakly holomorphic modular forms of weight $k$ for $\Gamma_{0}(4)$ whose Fourier coefficients satisfy the plus space condition.

We need the differential operator $\xi_{k}$ defined for any $k \in \mathbb{R}$ by

$$
\xi_{k} f(\tau)=2 \mathrm{i} y^{k} \overline{f_{\bar{\tau}}(\tau)}
$$

Clearly $\xi_{k} f=0$ if and only if $f$ is holomorphic. The operator $\xi$ is related to the weight $k$ Laplacian via

$$
-\xi_{2-k} \circ \xi_{k}=\Delta_{k}=-y^{2}\left(\partial_{x}^{2}+\partial_{y}^{2}\right)+\mathrm{i} y k\left(\partial_{x}+\mathrm{i} \partial_{Y}\right)
$$

When $k \in \frac{1}{2} \mathbb{Z}$ it is readily checked that if $f$ has weight $k$ for $\Gamma_{0}(4)$, then $\xi_{k} f$ has weight $2-k$ for $\Gamma_{0}$ (4) (see [2] for further results about the operator $\xi_{k}$.)

Lemma 3.2. Suppose $k \in \frac{1}{2}+\mathbb{Z}$ and that $g$ is holomorphic on $\mathcal{H}$ of weight $k$ for $\Gamma_{0}(4)$ whose Fourier expansion satisfies the plus space condition. Suppose that $h$ is a smooth function of weight $2-k$ for $\Gamma_{0}(4)$ whose Fourier expansion satisfies the plus space condition for weight $2-k$. Then, for $Y \geq 2$ we have

$$
\int_{\mathcal{F}_{4}(Y)} g(\tau) \overline{\xi_{2-k} h(\tau)} y^{k} \frac{\mathrm{d} x \mathrm{~d} y}{y^{2}}=\int_{-1 / 2+\mathrm{i} Y}^{1 / 2+\mathrm{i} Y}\left(g(\tau) h(\tau)+\frac{1}{2} g^{e}(\tau) h^{e}(\tau)+\frac{1}{2} g^{\mathrm{o}}(\tau) h^{\mathrm{o}}(\tau)\right) \mathrm{d} \tau .
$$

Proof. By (3.7) and the identity $\mathrm{d} \tau \mathrm{d} \bar{\tau}=2 \mathrm{i} \mathrm{d} x \mathrm{~d} y$ we have

$$
\int_{\mathcal{F}_{4}(Y)} g(\tau) \overline{\xi_{2-k} h(\tau)} y^{k} \frac{\mathrm{d} x \mathrm{~d} y}{y^{2}}=\int_{\mathcal{F}_{4}(Y)} g(\tau) h_{\bar{\tau}}(\tau) \mathrm{d} \tau \mathrm{d} \bar{\tau} .
$$

We now apply Green's theorem in the form

$$
\int_{\mathcal{F}_{4}(Y)} g(\tau) h_{\bar{\tau}}(\tau)+h(\tau) g_{\bar{\tau}}(\tau) \mathrm{d} \tau \mathrm{d} \bar{\tau}=-\int_{\partial \mathcal{F}_{4}(Y)} g(\tau) h(\tau) \mathrm{d} \tau
$$

in which the second term of the first integral vanishes as $g$ is holomorphic. Now $f=g h$ has weight 2 for $\Gamma_{0}(4)$ and so satisfies $\left.f\right|_{2} \gamma=f$ for all $\gamma \in \Gamma_{0}(4)$. Thus, by Lemma 3.1 
we have

$$
\int_{\mathcal{F}_{4}(Y)} g(\tau) \overline{\xi_{2-k} h(\tau)} y^{k} \frac{\mathrm{d} x \mathrm{~d} y}{y^{2}}=\int_{-1 / 2+\mathrm{i} Y}^{1 / 2+\mathrm{i} Y}\left(f(\tau)+\left.f\right|_{2} \sigma_{0}(\tau)+\left.f\right|_{2} \sigma_{1 / 2}(\tau)\right) \mathrm{d} \tau .
$$

We are reduced to proving the following identities, which follow easily from (3.6).

$$
\begin{gathered}
\left.(g h)\right|_{2} \sigma_{0}=\frac{1}{2} g^{e} h^{e}, \\
\left.(g h)\right|_{2} \sigma_{1 / 2}=\frac{1}{2} g^{o} h^{o} .
\end{gathered}
$$

\section{Inner Products}

First we prove Theorem 2.1. In fact, we have a more general result. Recall the general Hurwitz number $h^{*}(d)$ defined in (2.3). Theorem 2.1 is a special case of the following result.

Proposition 4.1. Suppose that $d$ is a positive non-square discriminant. Then

$$
\left\langle g_{d}, g_{0}\right\rangle=-\frac{3}{4} d^{-1 / 2} h^{*}(d)
$$

Proof. Recall the function $g_{0}$, which was defined in (2.5). It was shown in [3] that there is a real analytic function $h(\tau)$ having weight $\frac{1}{2}$ for $\Gamma_{0}(4)$ with

$$
\xi_{1 / 2} h(\tau)=-2 g_{0}(\tau)
$$

Let $\mathcal{P}$ denote the set of all positive non-square discriminants and $\mathcal{P}^{c}$ the rest of the discriminants. The Fourier expansion of $h$ can be written as

$$
h(\tau)=\sum_{n \in \mathcal{P}} n^{-1 / 2} h^{*}(n) q^{n}+\sum_{n \in \mathcal{P}^{c}} a(n, y) e(n x)
$$

where the function defined by the second sum is $\ll y^{1 / 2}$ for $y \geq 2$. By Lemma 3.2 we have

$$
\left\langle g_{d}, g_{0}\right\rangle=-\frac{1}{2} \lim _{Y \rightarrow \infty} \int_{-1 / 2+\mathrm{i} Y}^{1 / 2+\mathrm{i} Y}\left(g_{d}(\tau) h(\tau)+\frac{1}{2} g_{d}^{e}(\tau) h^{e}(\tau)+\frac{1}{2} g_{d}^{\mathrm{o}}(\tau) h^{\mathrm{o}}(\tau)\right) \mathrm{d} \tau
$$

Using the Fourier expansions of $h$ and $g_{d}$ and the fact that $g_{d}(\tau)=q^{-d}+O(q)$ from (2.7) when $d$ is not a square, we see that only the constant terms of the products can 
contribute to the last integral. Now we deduce Proposition 4.1 upon using (3.5) to check that we get a non-zero contribution from $g_{d}^{e}(\tau) h^{e}(\tau)$ only when $d$ is even while we get one from $g_{d}^{\mathrm{o}}(\tau) h^{\mathrm{o}}(\tau)$ only when $d$ is odd.

It is easy to check that

$$
\xi_{3 / 2} g_{0}(\tau)=-\frac{1}{16 \pi} \theta(\tau)
$$

Thus by (3.8) and (4.1) we see that $h$ satisfies the inhomogeneous equation

$$
\Delta_{1 / 2} h(\tau)=-\frac{1}{8 \pi} \theta(\tau)
$$

In order to prove Theorem 2.2 we need to employ weakly harmonic modular forms of weight $\frac{1}{2}$ for $\Gamma_{0}(4)$. For background on their theory we refer to [3] and the references there, especially [2]. Suppose that $f$ is a real analytic function on $\mathcal{H}$ of weight $k$ for $\Gamma_{0}(4)$ that is harmonic on $\mathcal{H}$ in the sense that

$$
\Delta_{k} f=0 \text {. }
$$

Such an $f$ will have a Fourier expansion at i $\infty$ each of whose terms has at most linearly exponential growth. Such an $f$ is called weakly harmonic if it has only finitely many such terms. If the Fourier expansion satisfies the plus space condition then by (3.6) its growth in the other cusps is at most linearly exponential. The space of all such forms is denoted by $H_{k}^{!}$. It is clear from (3.8) that $M_{k}^{!} \subset H_{k}^{!}$.

It follows from [3, Theorem 1] that $H_{1 / 2}^{!}$has a natural basis $\left\{h_{d}\right\}_{d \equiv 0,1(4)}$ where $\left\{h_{d}\right\}_{d \leq 0}$ is the Borcherds basis for $M_{1 / 2}^{!}$with $h_{d}(\tau)=q^{d}+\mathrm{O}(q)$ for each $d \leq 0$. For $d>0$ we have that $h_{d}$ satisfies $\xi_{1 / 2} h_{d}=2 d^{1 / 2} g_{d}$ and its Fourier expansion has the form

$$
h_{d}(\tau)=a_{d}(d, y) e(\mathrm{~d} x)-4 y^{1 / 2} \delta \square, d+\sum_{\substack{0<n=0,1(4) \\ n \neq d}} n^{-1 / 2} a_{d}(n) q^{n}+\sum_{\substack{n \equiv 0,1(4) \\ n<0}} a_{d}(n, y) e(n x),
$$

where $a_{d}(d, y) \sim 1 / 2 \pi(\mathrm{d} y)^{-1 / 2} \mathrm{e}^{2 \pi \mathrm{d} y}$ as $y \rightarrow \infty$ is the lone exponentially growing term and the function defined by the second sum is bounded for $y \geq 2$. An argument like that in the previous proof yields the following result. 
Proposition 4.2. Suppose that $n$ and $d$ are distinct positive discriminants, not both squares. Then

$$
\left\langle g_{n}, g_{d}\right\rangle=\frac{3}{4}(n d)^{-1 / 2} a_{d}(n)
$$

It was shown in [3] that $a_{d}(n)=a_{n}(d)$ so it follows that $\left\langle g_{n}, g_{d}\right\rangle \in \mathbb{R}$. If $d=n$ or both are squares, then the regularized inner product will not be finite. However, one can isolate the growing terms and subtract them in order to compute $a_{d}(n)$.

\section{Values of Modular Functions at Real Quadratic Numbers}

Now we apply more results of [3] to deduce Theorem 2.2. For any modular function $f \in \mathbb{C}[j]$ we define $f(w)$ for $w \in \mathcal{Q}(d)$ with $d>0$ not a square by

$$
f\left(\frac{-b+\sqrt{d}}{2 a}\right)=\frac{1}{2 R(d)} \int_{-\alpha}^{\alpha} f\left(-\frac{b}{2 a}+\frac{\mathrm{i} \sqrt{d}}{2|a|} \mathrm{e}^{\mathrm{i} \theta}\right) \frac{\mathrm{d} \theta}{\cos \theta}
$$

where $\alpha=2 \tan ^{-1}(u \sqrt{d} / t)$ and $t, u \in \mathbb{Z}^{+}$solve $t^{2}-\mathrm{d} u^{2}=4$ with $u$ minimal. If $w=(-b+$ $\sqrt{d}) / 2 a$, a computation shows that for $Q(x, y)=a x^{2}+b x y+c y^{2}$ we have

$$
R(d) f(w)=\int_{C_{Q}} f(\tau) \mathrm{d} \tau_{Q} \quad \text { where } \mathrm{d} \tau_{Q}=\frac{\sqrt{d}}{Q(\tau, 1)} \mathrm{d} \tau
$$

and $C_{Q}$ is any smooth curve from $z=-b / 2 a+\mathrm{i} \sqrt{d} / 2|a| \in \mathcal{H}$ to $g_{Q} z$, where

$$
g_{Q}= \pm\left(\begin{array}{cc}
\frac{t+b u}{2} & c u \\
-a u & \frac{t-b u}{2}
\end{array}\right)
$$

It follows from [3] that $f(\gamma w)=f(w)$ for all $\gamma \in \Gamma$. Observe that under this extension a constant function remains constant. It is well known that $\mathbb{C}[j]$, has a unique basis $\left\{j_{m}\right\}_{m \geq 0}$ of the form

$$
j_{m}(\tau)=q^{-m}+\sum_{n \geq 1} c_{m}(n) q^{n}
$$

This $j_{m}$ can be obtained from $j_{1}$ by applying the $m$ th Hecke operator $T_{m}$ or defined recursively. The following is a special case of the main result of [3]. 
Proposition 5.1. Suppose that $d$ and $d^{\prime}$ are distinct positive fundamental discriminants and that $m \geq 1$. Then

$$
\sum_{n \mid m} \chi_{d}\left(\frac{m}{n}\right) a_{d^{\prime}}\left(n^{2} d\right)=\frac{R(D)}{2 \pi} \sum_{w \in \Gamma \backslash \mathcal{Q}(D)} \chi(w) j_{m}(w),
$$

where $D=d d^{\prime}$ and $\chi$ is the associated genus character.

Theorem 2.2 now follows from Propositions 4.2 and 5.1 with $m=1$.

\section{Funding}

This work is partially supported by the National Science Foundation grant number DMS-0355564 (W.D.), SNF grant number is 200021-119796 (O.I.) and OTKA grants K 72731 and NK 81203 (A.T.).

\section{References}

[1] Borcherds, R. E. "Automorphic forms with singularities on Grassmannians." Inventiones Mathematicae 132, no. 3 (1998): 491-562.

[2] Bruinier, J. H. and J. Funke. “On two geometric theta lifts." Duke Mathematical Journal 125, no. 1 (2004): 45-90.

[3] Duke, W., Ö. Imamoḡlu, and A. Toth. "Cycle integrals of the $j$-function and mock modular forms." Annals of Mathematics, to appear.

[4] Hirzebruch, F. and D. Zagier. "Intersection numbers of curves on Hilbert modular surfaces and modular forms of Nebentypus." Inventiones Mathematicae 36 (1976): 57-113.

[5] Kaneko, M. "Observations on the 'values' of the elliptic modular function $j(\tau)$ at real quadratics." Kyushu J. Math. 63, no. 2 (2009): 353-64.

[6] Kohnen, W. and D. Zagier. "Values of $L$-series of modular forms at the center of the critical strip." Inventiones Mathematicae 64, no. 2 (1981): 175-98.

[7] Shimura, G. "On modular forms of half integral weight." Annals of Mathematics (2) 97 (1973): 440-81.

[8] Weber, H. Complexe Multiplication. Encykl. d. math. Wiss. 1 (1900): 716-32.

[9] Zagier, D. “Nombres de classes et formes modulaires de poids 3/2." (French) Comptes Rendus Mathématique Académie des Sciences Paris A-B 281, no. 21 (1975): Ai, A883-6.

[10] Zagier, D. "Traces of singular moduli." Motives, polylogarithms and Hodge theory, Part I (Irvine, CA, 1998), 211-44. International Press Lecture Series 3, I. Somerville, MA: International Press, 2002. 